\title{
Spatial Correlation of Particulate Matter Pollution and Death Rate of COVID-19
}

Ye Yao ${ }^{\dagger}$, Ph.D., Fudan University, Shanghai China

Jinhua Pan ${ }^{\dagger}$, M.Sc., Fudan University, Shanghai China

Weidong Wang ${ }^{\dagger}$, B.Med., Fudan University, Shanghai China

Zhixi Liu ${ }^{\dagger}$, B.Med., Fudan University, Shanghai China

Haidong Kan, Ph.D. \& M.D., Fudan University, Shanghai China

Xia Meng ${ }^{*}$, Ph.D., Fudan University, Shanghai China

Weibing Wang*, Ph.D. \& M.D., Fudan University, Shanghai China

${ }^{\dagger}$ Dr. Yao, Ms. Pan, Mr. Wang and Ms. Liu contributed equally to this letter.

* Corresponding authors:

Dr. Weibing Wang, School of Public Health, Fudan University, Shanghai

200032, China, Email: wwb@fudan.edu.cn

Dr. Xia Meng, School of Public Health, Fudan University, Shanghai

200032, China, Email: mengxia@ @udan.edu.cn

Word account:1116 
medRxiv preprint doi: https://doi.org/10.1101/2020.04.07.20052142; this version posted April 10, 2020. The copyright holder for this preprint (which was not certified by peer review) is the author/funder, who has granted medRxiv a license to display the preprint in perpetuity.

\author{
It is made available under a CC-BY-NC-ND 4.0 International license.
}




\begin{abstract}
The Coronavirus (COVID-19) epidemic, which was first reported in December 2019 in Wuhan, China, has caused 3,314 death as of March 31, 2020 in China. This study aimed to investigate the spatial associations of daily particulate matter (PM) concentrations with death rate of COVID-19 in China. We conducted a cross-sectional analysis to examine the spatial associations of daily PM2.5 and PM10 concentrations with death rate of COVID-19 in China through multiple linear regression method. We found that COVID-19 held higher death rates with increasing concentration of PM2.5 and PM10 levels in the spatial scale, which may affect the process of patients developed from mild to severe and finally influence the prognosis of COVID-19 patients.
\end{abstract}

\title{
Introduction
}

SARS-CoV-2 is a new emerge of coronavirus that poses immense challenges to global health and the economy. As of April 3, 2020, a total of 1,042,588 COVID-19 cases have been confirmed in 199 countries worldwide. In China, a total of 82,772 confirmed cases and 3,327 deaths were recorded. World Health Origination (WHO) announced on March 11 that the assessment of the COVID-19 outbreak has been a global pandemic. 
The hypothesis that air pollution can act both as a vector of the infection and as a worsening factor of the health impact of the pandemic in progress has been raised recently. Short-term exposure to ambient particulate matter (PM) pollution has been associated with increasing risks of mortality and morbidity of cardiopulmonary diseases worldwide $^{1,2}$, and the adverse effects are significantly higher for people who are older or with underlying medical conditions ${ }^{3}$, among which the fatality of COVID-19 is also higher. While previous studies suggested that short-term exposure to PM could aggravate respiratory symptoms and increase the emergency visits of patients with asthma and chronic obstructive pulmonary disease (COPD) ${ }^{4}$, the European Public Health Alliance have recently reported that air pollution is likely to increase the death rate of SARS-CoV-2 in cities ${ }^{5}$. Air pollution, especially PM pollution, was approved to be also positively associated case fatality of severe acute respiratory syndrome (SARS) in China ${ }^{6}$.

However, few studies have estimated and quantified the short-term effects of air pollutants on early deaths from COVID-19. While the extent of correlation is less known, this study aimed to investigate the association between death rate of COVID-19 and $\mathrm{PM}_{2.5}$ and $\mathrm{PM}_{10}$ pollutions in a spatial scale.

\section{Methods}


We conducted a cross-sectional analysis to examine the spatial associations of daily $\mathrm{PM}_{2.5}$ and $\mathrm{PM}_{10}$ concentrations with death rate of COVID-19 in China through multiple linear regression method. The COVID-19 confirmed cases and deaths information in China was collected from the National Health Commission and the Provincial Health Commissions of China. We calculated death rate, which was defined as cumulative death counts divided by cumulative confirmed cases, for 49 cities including Wuhan, other 15 cities inside Hubei and 33 cities outside Hubei, with no less than 100 cases as of March 22 (few confirmed cases and deaths were reported afterwards). Daily $\mathrm{PM}_{2.5}$ and $\mathrm{PM}_{10}$ data were collected from the National Urban Air Quality Publishing Platform (http://106.37.208.233:20035/), which is administered by China's Ministry of Environmental Protection. And meteorological data including daily mean temperature and relative humidity were collected from the China Meteorological Data Sharing Service System. Gross Domestic Product (GDP) per capita, hospital beds and population size were obtained from statistical yearbook of each province.

\section{Results}

As of March 22, the mean death rate of COVID-19 was 1.7\% among the 49 cities in China, with a range of $0.0 \%-5.0 \%$. And the mean \pm standard deviation and range were $\left(51.2 \pm 20.9,20.8-96.9 \mu \mathrm{g} / \mathrm{m}^{3} \quad \&\right.$ 
62.1 $22.6, \quad 30.8-115.1 \mu \mathrm{g} / \mathrm{m}^{3}$ ) for $\mathrm{PM}_{2.5} \& \mathrm{PM}_{10}$. There were no significant $\mathrm{PM}_{2.5}$ or $\mathrm{PM}_{10}$ differences between cities outside and inside Hubei $(\mathrm{t}=0.48, p=0.63 \& \mathrm{t}=0.63, p=0.53)$. Death rate of cities inside Hubei except Wuhan was significantly lower than that of Wuhan $(\mathrm{t}=6.70$, $p=5.0 \times 10^{-5}$ ), while death rate of cities outside Hubei was even lower than those inside Hubei $\left(\mathrm{t}=8.26, p=2.6 \times 10^{-10}\right)$.

After adjustment for temperature, relative humidity, GDP per capita and hospital beds per capita, COVID-19 death rate was positively associated with concentration of $\mathrm{PM}_{2.5}$ (Figure 1A, $\chi^{2}=13.10, \mathrm{p}=0.011$ ) and $\mathrm{PM}_{10}$ (Figure 1B, $\left.\chi^{2}=12.38, \mathrm{p}=0.015\right)$, suggesting that COVID-19 held higher death rates with increasing concentration of $\mathrm{PM}_{2.5}$ and $\mathrm{PM}_{10}$ levels in the spatial scale. In addition, we did not find significance in the association between GDP per capita, hospital beds per capita, temperature or relative humanity and COVID-19 death rate $\left(\chi^{2}=6.02, p=0.20 \&\right.$ $\left.\chi^{2}=3.68, p=0.45 \& \chi^{2}=3.76, p=0.44 \& \chi^{2}=7.21, p=0.13\right)$.

\section{Discussions}

Our results demonstrate that the death rate of coronavirus disease (COVID-19) has a strong association with $\mathrm{PM}_{2.5}$ and $\mathrm{PM}_{10}$, whether in Hubei province or other cities in China. Our results are consistent with previous studies of SARS ${ }^{6}$. COVID-19 is caused by SARS-CoV-2, which shares $79.6 \%$ sequence identity to SARS-CoV and has the same cell entry receptor - angiotensin converting enzyme II - as SARS-CoV. Limited 
medRxiv preprint doi: https://doi.org/10.1101/2020.04.07.20052142; this version posted April 10, 2020. The copyright holder for this preprint

studies have researched on the associations between air pollution and SARS which only lasted for half year in 2003 and only a few Chinese cities had adequate death data for analysis. A previous one reported that moderate air pollution index (APIs), which was dominated by $\mathrm{PM}_{10}$ in China in 2003, had an $84 \%$ increased risk of dying from SARS compared to those from regions with low APIs, and SARS patients from regions with high APIs were twice as likely to die from SARS compared to those from regions with low APIs. Other previous studies demonstrated that long-term or short-term exposure to $\mathrm{PM}_{10}$ and $\mathrm{PM}_{2.5}$ might damage the lung functions ${ }^{7-9}$. The mechanism of the effects from PM exposure on respiratory outcomes might be caused by activation of inflammatory pathways in the small airways in response to PMs, leading to the recruitment of inflammatory cells ${ }^{10}$. These biological mechanisms might potentially influence the prognosis of COVID-19 patients.

Considering the fact that the patients died from COVID-19 are likely to be critically ill, most of them might stay in Intensive Care Unit (ICU) for treatment. We speculate that the effects of $\mathrm{PM}_{2.5}$ and $\mathrm{PM}_{10}$ on death mainly affect the progress of patients from mild to severe and prognosis. There is a need to increase our efforts in the control of air pollutant emissions, especially regarding the situation that the COVID-19 epidemics may come back at any time.

The study was limited to a short period of season with less variation 
of air pollution. However, the correlation of trends of death and air pollution is quite convincing. Given the ecological nature of study, other city-level factors, such as implementation ability of COVID-19 control policy, urbanization rate, and availability of medical resources, may affect the death rate of COVID-19 and confound our findings, however, we control the GDP per capita and hospital beds per capita increased the credibility of the results.

\section{Author contributions}

Dr. Ye Yao, Jinhua Pan, Weidong Wang and Zhixi Liu contributed equally.

Dr. Weibing Wang and Dr. Xia Meng contributed equally.

Concept and design: Ye Yao, Xia Meng, and Weibing Wang.

Acquisition, analysis, or interpretation of data: Ye Yao, Jinhua Pan, Zhixi Liu, Haidong Kan and Weidong Wang.

Drafting of the manuscript: Ye Yao, Jinhua Pan, Zhixi Liu.

Critical revision of the manuscript for important intellectual content: Haidong Kan, Xia Meng and Weibing Wang.

Statistical analysis: Ye Yao and Xia Meng.

\section{Competing interests}

The authors declare no competing interests.

\section{Acknowledgements}


This study was sponsored by the Bill \& Melinda Gates Foundation (Grant No.

OPP1216424).

\section{References}

1. Chen R, Yin P, Meng X, et al. Fine Particulate Air Pollution and Daily Mortality. A Nationwide Analysis in 272 Chinese Cities. Am J Respir Crit Care Med. 2017;196(1):73-81.

2. Samet JM, Zeger SL, Dominici F, et al. The National Morbidity, Mortality, and Air Pollution Study. Part II: Morbidity and mortality from air pollution in the United States. Res Rep Health Eff Inst. 2000;94(Pt 2):5-70; discussion 71-79.

3. Zeka A, Zanobetti A, Schwartz J. Individual-level modifiers of the effects of particulate matter on daily mortality. Am J Epidemiol. 2006;163(9):849-859.

4. EPA. Integrated Science Assessments (ISAs). 2020; https://www.epa.gov/isa.

5. Alliance EPH. Coronavirus threat greater for polluted cities. 2020; https://epha.org/coronavirus-threat-greater-for-polluted-cities/.

6. Cui $Y$, Zhang ZF, Froines J, et al. Air pollution and case fatality of SARS in the People's Republic of China: an ecologic study. Environ Health. 2003;2(1):15.

7. Doiron D, de Hoogh K, Probst-Hensch N, et al. Air pollution, lung function and COPD: results from the population-based UK Biobank study. Eur Respir J. 2019;54(1).

8. Bloemsma LD, Hoek G, Smit LAM. Panel studies of air pollution in patients with COPD: Systematic review and meta-analysis. Environ Res. 2016;151:458-468.

9. Rice MB, Ljungman PL, Wilker EH, et al. Short-term exposure to air pollution and lung function in the Framingham Heart Study. Am J Respir Crit Care Med. 
10. Kelly FJ, Fussell JC. Air pollution and airway disease. Clin Exp Allergy. 2011;41(8):1059-1071.

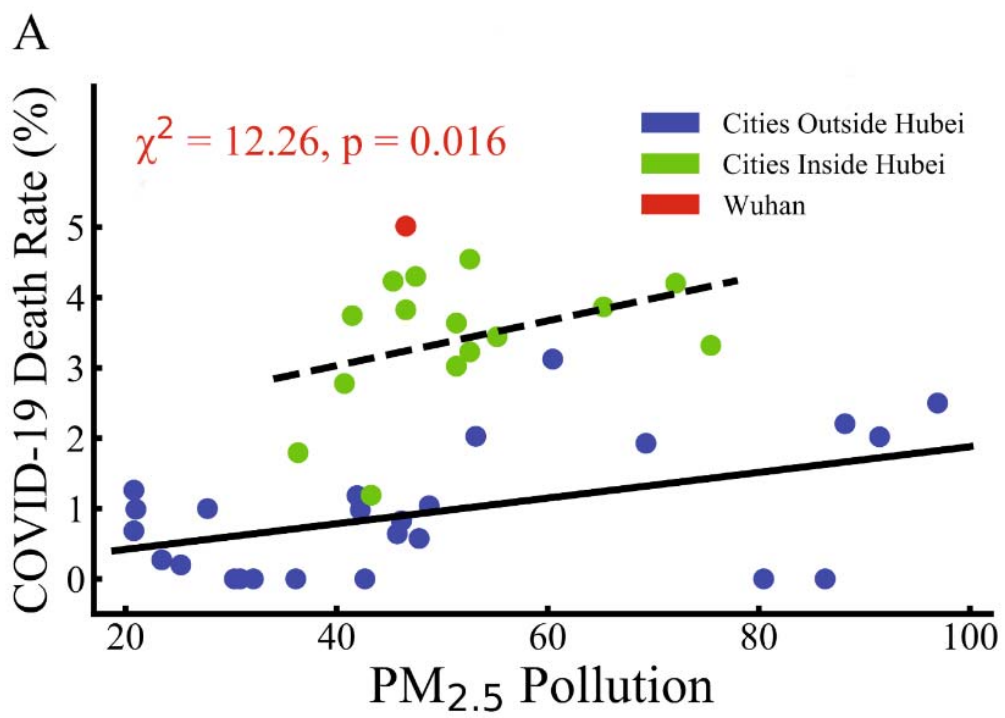

$\mathrm{B}$

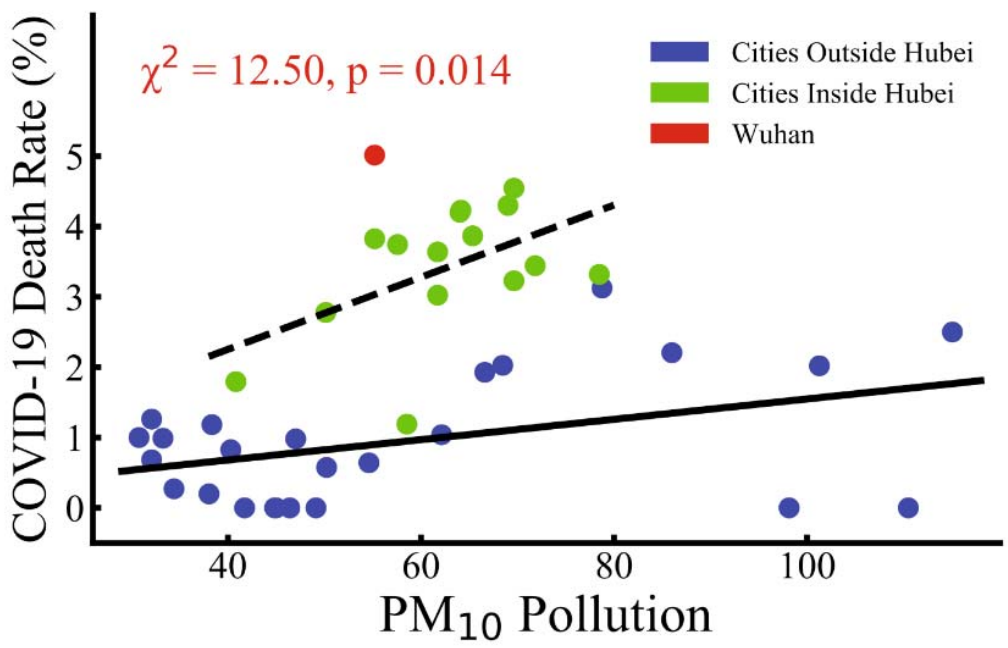

Figure 1 Death Rate Versus $\mathrm{PM}_{2.5} \& \mathrm{PM}_{10}$ pollution.

Figure 1A: Death Rate was positively associated (Meta $\chi^{2}=13.10$, $\mathrm{p}=0.011$ ) with $\mathrm{PM}_{2.5}$ in cities outside Hubei (blue points, $\mathrm{r}=0.56, \mathrm{p}=0.005$ ) 
and those inside Hubei except Wuhan (green points, $\mathrm{r}=0.33, \mathrm{p}=0.26$ ) pollution. GDP per capita and hospital beds per capita effects were removed during statistical analysis. Figure 1B: Death Rate was positively associated (Meta $\chi^{2}=12.38, \mathrm{p}=0.015$ ) with $\mathrm{PM}_{10}$ in cities outside Hubei (blue points, $\mathrm{r}=0.48, \mathrm{p}=0.019$ ) and those inside Hubei except Wuhan (green points, $\mathrm{r}=0.47, \mathrm{p}=0.11$ ) pollution. GDP per capita and hospital beds per capita effects were removed during statistical analysis. 